for CBT seem to be an example of the latter practice being applied to the results of multiple meta-analyses.

1 Taylor M, Perera U. NICE CG178 Psychosis and Schizophrenia in Adults: Treatment and Management - an evidence-based guideline? Br J Psychiatry 2015; 206: 357-9.

2 National Institute for Health and Care Excellence. Psychosis and Schizophrenia in Adults: Treatment and Management (CG 178). NICE, 2014.

3 Chan AW. Bias, spin, and misreporting: time for full access to trial protocols and results. PLOS Med 2008; 5: e230.

P. J. McKenna, Germanes Hospitalàries Research Foundation and CIBERSAM, Spain. Email: mckennapeter1@gmail.com; K. R. Laws, School of Life and Medical Sciences, University of Hertfordshire, UK; S. Jauhar, Department of Psychosis Studies, Institute of Psychiatry, London, UK

doi: 10.1192/bjp.207.6.560a

Authors' reply: We thank Dr McKenna (and colleagues) for his interest in our editorial, and respect his long record of research into schizophrenia. His point about the authors of influential national clinical guidelines such as NICE, the British Association for Psychopharmacology (BAP) and the Scottish Intercollegiate Guidelines Network (SIGN) needing to take negative evidence into account is well made, and analogous to the AllTrials movement in pharmacotherapeutics. Schizophrenia is such a common and potentially devastating illness that it is incumbent on mental health professionals such as psychologists and psychiatrists to work together to deliver best-evidenced treatments.

Mark Taylor, Royal Edinburgh Hospital, Edinburgh, UK, and University of Queensland Brisbane, Australia. Email: marktaylor2@nhs.net; Udayanga Perera, District Genera Hospital, Monaragala, Sri Lanka

doi: 10.1192/bjp.207.6.561

\section{Does previous experience of antidepressants form the expectations necessary for a placebo response?}

Leuchter et al's ${ }^{1}$ findings extend the current understanding of the placebo response and raise important questions regarding the design of antidepressant trials. An important finding was that expectation of medication effectiveness predicted treatment response in the placebo group only, which suggests that expectations of treatment benefit are required for a placebo response.

It is thought that the placebo response results from an interaction between expectations and learning. ${ }^{2}$ In studies of placebo analgesia, experimental paradigms often involve a conditioning procedure to induce an expectation of benefit from treatment. One widely used paradigm involves thermal pain stimulation and application of an inert cream. Following application of the cream, the thermal energy is reduced to non-painful levels to condition the participant to believe the cream has analgesic properties. Subsequently, laser stimulation continues at painful levels, and participants report the stimulation as less painful. ${ }^{3-6}$ The implication is that an expectation of analgesia, induced by exposure to the cream's 'analgesic' properties, results in a placebo response. $^{3}$ Learning to expect an effect has also been shown to influence emotional processing. Petrovic et $\mathrm{al}^{7}$ measured responses to aversive pictures in healthy volunteers following administration of placebo 'anxiolytic' medication and its reversal, and found that participants reported aversive pictures as less distressing when they thought they had received anxiolytic medication, and more distressing when they believed this had been reversed. This result shows that a learned expectation, induced through exposure to a medication, can cause changes in emotional processing.

In the study reported by Leuchter $e t a l,{ }^{1}$ there was a relationship between expectation of benefit and treatment response in the placebo group. However, these patients did not undergo a conditioning procedure to induce an expectation of benefit. What caused these patients to expect a benefit? Could the therapeutic environment and consent process for starting an antidepressant engender a powerful expectation of benefit on its own? Or does this expectation come from previous experience of benefit from antidepressant treatment? The data from this study suggest the latter, as the expectations seemed to be formed at the time of enrolment. We could perhaps answer this question more fully through assessment of the relationship between previous response to antidepressant treatment and placebo response in this trial. It is possible that more patients in the placebo group had previously benefitted from treatment than in the medication group, and if this were so, it would lend support to the idea that previous experience of benefit from antidepressant treatment could cause a placebo antidepressant response. This could be an important consideration in future antidepressant drug trials.

1 Leuchter AF, Hunter AM, Tartter M, Cook IA. Role of pill-taking, expectation and therapeutic alliance in the placebo response in clinical trials for major depression. Br J Psychiatry 2014; 205: 443-9.

2 Benedetti F, Carlino E, Pollo A. How placebos change the patient's brain. Neuropsychopharmacology 2011; 36: 339-54.

3 Price DD, Milling LS, Kirsch I, Duff A, Montgomery GH, Nicholls SS. An analysis of factors that contribute to the magnitude of placebo analgesia in an experimental paradigm. Pain 1999; 83: 147-56.

4 Morton DL, Brown CA, Watson A, El-Deredy W, Jones AKP. Cognitive changes as a result of a single exposure to placebo. Neuropsychologia 2010; 48: 1958-64.

5 Huneke NTM, Brown CA, Burford E, Watson A, Trujillo-Barreto NJ, El-Deredy $W$, et al. Experimental placebo analgesia changes resting-state alpha oscillations. PLOS One 2013; 8: e78278.

6 Watson A, El-Deredy W, Vogt BA, Jones AKP. Placebo analgesia is not due to compliance or habituation: EEG and behavioural evidence. Neuroreport 2007; 18: 771-5.

7 Petrovic $\mathrm{P}$, Dietrich $\mathrm{T}$, Fransson $\mathrm{P}$, Andersson J, Carlsson $\mathrm{K}$, Ingvar $\mathrm{M}$. Placebo in emotional processing-induced expectations of anxiety relief activate a generalized modulatory network. Neuron 2005; 46: 957-69.

Nathan T. M. Huneke, Psychiatry ACFST1, University of Southampton, UK. Email: nathan.huneke@doctors.org.uk; David S. Baldwin, Professor of Psychiatry and Head of the Mental Health Group, University of Southampton, UK

doi: $10.1192 /$ bjp.207.6.561a

Authors' reply: Huneke \& Baldwin raise important points regarding the interpretation of our study results and the relationship of our findings to the broader placebo literature. It is challenging to compare the results from our study with the literature cited by them. As they note, studies of placebo analgesia generally are performed in healthy volunteers not being treated for a chronic illness. Such studies examine the placebo effect, namely the relief of transient, experimentally induced symptoms during manipulation of expectations. By contrast, our study examined placebo response, which involves relief of naturally occurring symptoms of a chronic illness (in this case major depressive disorder, or MDD) within the context of a clinical trial. Because patients with MDD have long courses of illness and treatment, they commonly enter treatment studies with pre-existing expectations and beliefs, and our participants had indeed formed expectations about medications at the time of study enrolment. We concluded that these expectations were probably formed by factors external to the study, and speculated on the role that 
external social factors (such as direct-to-consumer advertising) might have had in forming positive medication expectations.

Huneke \& Baldwin raise the point that our participants' medication expectations, which predicted placebo response, may have been formed, at least in part, by the consent process and initial exposure to the study environment. Participants consented and had their introduction to study personnel prior to rating their expectations of improvement. Although we do not know to what extent medication expectations might have been influenced by this initial exposure, a significant effect is unlikely. Participants rated the degree to which they expected that treatment in general, and medication in particular, would be helpful in relieving their depression. If participants' initial exposure to the study milieu shaped expectations, it would be expected to influence both medication and general treatment expectations. Yet, only participants' ratings of medication expectations predicted response to placebo. The selective relationship between medication, but not general, treatment expectations and placebo outcome suggests the influence of a process outside of the study milieu.

We agree with Huneke \& Baldwin that it would be instructive to learn more about participants' previous experiences with antidepressant treatment and how this might affect current medication expectations, as well as the likelihood of placebo response. In this regard, we recently examined the potential role of prior antidepressant treatment and placebo treatment response in these same participants. ${ }^{1}$ Self-report data collected from a subset of participants from the parent study revealed that previous experience with antidepressant medication was significantly associated with poorer response to placebo. Interestingly, among those who had received prior antidepressant treatment, their self-report of response to prior treatment was not significantly related to expectations in the current trial or to placebo outcome. This finding suggests that antidepressant-experienced participants may show classic conditioning effects, consistent with our previously reported findings. ${ }^{2}$ The finding that prior antidepressant exposure, regardless of response, predicts placebo outcome is worthy of future study.

1 Hunter AM, Cook IA, Tartter M, Sharma SK, Disse GD, Leuchter AF. Antidepressant treatment history and drug-placebo separation in a placebocontrolled trial in major depressive disorder. Psychopharmacology (Berl) 2015; 232: 3833-40.

2 Hunter AM, Cook IA, Abrams M, Leuchter AF. Neurophysiologic effects of repeated exposure to antidepressant medication: are brain functional changes during antidepressant administration influenced by learning processes? Med Hypotheses 2013; 81: 1004-11.

Andrew F. Leuchter, Semel Institute for Neuroscience and Human Behavior at UCLA, Director of Neuromodulation Division, Los Angeles, USA. Email: afl@ucla.edu; Aimee M. Hunter, Molly Tartter, Ian A. Cook, UCLA, LOS Angeles, USA

doi: 10.1192/bjp.207.6.561b
1 The unpredictable nature of suicide in participants with no or low suicidality is of major concern, especially for ethical reasons.

2 Either obtaining a detail clinical history (medical and psychiatric) and mental state examination by mental health professionals or using a screening instrument like the Composite International Diagnostic Interview ${ }^{2}$ prior to the laboratory experiment would have helped in ruling out other psychiatric disorders as a part of exclusion criteria and would have served the purpose adequately.

3 The possibility of unreliable responses among participants with ongoing psychotic illnesses like schizophrenia in all the scales cannot be ruled out completely.

4 The Erlanger Depression Scale ${ }^{3}$ consists of 9 statements on a printed form with 5 possible answers ranging from 'accurate' to 'not true', and has been wrongly described as having 8 items rated on a scale from 0 (completely wrong) to 4 (exactly right).

5 The Reasons for Living Scale, ${ }^{4}$ which has 72 items, has been wrongly described as having 48 items. It is only in the revised scale that 24 out of 72 items were dropped because of ambiguous factor loading.

6 Reason for excluding other subscales of the World Assumptions Scale ${ }^{5}$ like 'justice', 'benevolence of people', 'randomness' and 'self-worth' is not mentioned.

7 Other factors like camera positioning, ${ }^{6}$ audio quality, lighting, and special effects studied for stimulating cue-induced craving in substance use disorders, have a qualitative role in predicting outcome and not only how the film ends.

1 Till B, Strauss M, Sonneck G, Niederkrotenthaler T. Determining the effects of films with suicidal content: a laboratory experiment. Br J Psychiatry 2015; 207: 72-8.

2 Kessler RC, Ustün TB. The World Mental Health (WMH) Survey Initiative version of the World Health Organization (WHO) Composite International Diagnostic Interview (CIDI). Int J Meth Psychiatr Res 2004; 13: 93-121.

3 Lehrl S, Gallwitz A. Erlanger Depression Scale (EDS) [in German]. Vless, 1983.

4 Linehan MM, Goodstein JL, Nielsen SL, Chiles JA. Reasons for staying alive when you are thinking of killing yourself: the Reasons for Living Inventory. J Consult Clin Psychol 1983; 51: 276-86.

5 Janoff-Bulman R. Assumptive worlds and the stress of traumatic events: applications of the schema construct. Soc Cogn 1989; 7: 113-36.

6 Brody AL, Mandelkern MA, Lee G, Smith E, Sadeghi M, Saxena S, et al. Attenuation of cue-induced cigarette craving and anterior cingulate cortex activation in bupropion-treated smokers: a preliminary study. Psychiatry Res 2004; 130: 269-81.

Shailesh Jha, Psychiatrist, Senior Resident, Institute of Human Behaviour and Allied Sciences (IHBAS), Delhi, India. Email: dr.shaileshk.jha@gmail.com; Rajesh Kumar Psychiatrist, IHBAS, Delhi, India

doi: 10.1192/bjp.207.6.562

Authors' reply: Regarding the questionnaires discussed by Drs Jha \& Kumar, we want to clarify some statements regarding some of the measures of our study. As they correctly pointed out, the Erlanger Depression Scale ${ }^{1}$ consists of 9 items, but only 8 of these items are used to calculate the score for depression, and the first item of the scale is a 'warm-up' item used for introduction to the scale. Further, the 48 -item scale by Linehan and colleagues ${ }^{2}$ is commonly referred to as the Reasons for Living Inventory, ${ }^{3-5}$ even though earlier versions of this scale may exist.

We agree that factors other than the outcome of the suicidal crisis portrayed in the films (e.g. camera positioning, audio quality, lighting, special effects) might have determined the impact 